Revista de Psicología Vol. 33 (2), 2015 (ISSN 0254-9247)

\title{
Dependencia emocional en mujeres víctimas de violencia de pareja
}

\author{
Jesús Joel Aiquipa Tello ${ }^{1}$ \\ Universidad Nacional Mayor de San Marcos
}

El siguiente estudio tiene como objetivo identificar la relación entre la dependencia emocional y la violencia de pareja en una muestra conformada por mujeres usuarias del servicio de psicología de un establecimiento de salud nacional. Para esto, se sigue un enfoque cuantitativo y se utiliza una estrategia asociativa comparativa. La muestra estuvo constituida por dos grupos de mujeres, mujeres víctimas de violencia de pareja (25 usuarias) y mujeres que no fueron víctimas de violencia de pareja (26 usuarias). Los resultados sugieren que existe relación estadísticamente significativa entre las variables de estudio, puesto que se encontró diferencias entre los dos grupos de estudio y se halló que el valor que asumió el coeficiente de relación entre las variables fue altamente significativo.

Palabras clave: violencia de pareja, dependencia emocional, factor de riesgo.

\section{Emotional dependence of female victims of intimate partnership violence}

The following study aims to identify the relationship between emotional dependency and intimate partner violence in a sample of women who utilize the psychology service of a national health establishment. A quantitative approach is followed with a comparative associative strategy. The sample consisted of two groups of women, female victims of intimate partner violence ( 25 women) and women who were not victims of intimate partner violence (26 women). The results suggest that there is a statistically significant relationship between the variables of study, since differences between the two study groups was found and it was found that the value assumed correlation coefficient between variables was highly significant. Keywords: Intimate partner violence, emotional dependency, risk factor.

1 Psicólogo clínico (UNMSM), es asesor de investigación en la Asociación Vida Mujer y docente en la Universidad Inca Garcilaso de la Vega. Autor del Inventario de Dependencia Emocional-IDE. Dirección Postal: Jr. Sucre 224, Lima 31. Contacto: aitejjiii@hotmail.com 
La violencia hacia la mujer representa tanto una violación de sus derechos humanos como un problema de salud pública (GarcíaMoreno, Henrica, Watts, Ellsberg \& Heise, 2013). Asimismo, la violencia a la mujer ejercida por su pareja varón es la más frecuente respecto a los distintos contextos de violencia a las mujeres reportados. Esta situación se produce en todos los países, en todas las culturas y en todos los niveles sociales sin excepción (García-Moreno, Henrica, Watts, Ellsberg \& Heise, 2005).

Esta forma de violencia contra la mujer ha recibido distintas denominaciones según los diferentes investigadores, enfoques o idiomas, adoptando características también distintas. Así, ha sido denominada violencia doméstica, violencia intrafamiliar, violencia conyugal, violación matrimonial, violencia con la pareja íntima, etc. Para efectos del siguiente estudio se utilizará el término violencia de pareja (VIP) para describir específicamente la violencia a la mujer por parte de su pareja varón, sin importar el tiempo de relación o la formalidad social que adopte dicha relación (cónyuges, enamorados, novios, etc.). Además, se adoptará la definición propuesta por Heise y García-Moreno (2003), desarrollada y ampliada por Butchart, García-Moreno y Mikton (2011), quienes consideran a la violencia de pareja como un tipo de violencia interpersonal y la definen como cualquier comportamiento que dentro de una relación íntima o de pareja, causa daño físico, psíquico o sexual. Este comportamiento, como los autores manifiestan, incluye agresiones físicas (abofetear, golpear con los puños o con cualquier parte del cuerpo, golpes con objetos, empujones, estrangulamiento, etc.), relaciones sexuales forzadas, y otras formas de coacción sexual, maltrato psicológico (intimidación, denigración, humillación constantes, insultos, gritos, indiferencia, etc.), diversos comportamientos dominantes (aislarla de su familia y amistades, vigilar sus movimientos, restringir su acceso a la información o asistencia, etc.) e incluso la muerte. 
Así, el estudio publicado en 2013 por la Organización Mundial de la Salud (OMS, citado en García-Moreno et al., 2013) revela que la violencia de pareja es el tipo más común de violencia contra la mujer, ya que afecta al $30 \%$ de las mujeres en todo el mundo, llegando esta cifra hasta el $38 \%$ en algunas regiones. Se indica también que el 38\% del número total de homicidios femeninos se debe a la violencia conyugal, cifra por supuesto muy preocupante. Este mismo estudio indica que en las Américas, el 29.8\% de las mujeres han sido víctimas de violencia física y/o sexual ejercida por parte de su pareja.

En otro estudio internacional, Bott, Guedes, Goodwin y Adams (2014) realizaron un análisis comparativo de los datos sobre la violencia contra las mujeres por parte de su esposo/compañero, datos provenientes de encuestas nacionales de base poblacional, recogidos entre el 2003 y el 2009 en 12 países de América Latina y el Caribe. Se encontró, entre otros resultados, que la violencia contra las mujeres está generalizada en todos los países estudiados, aunque la prevalencia varía según el entorno. Asimismo, se halló que entre la cuarta parte y la mitad de las mujeres informaron haber sufrido alguna vez este tipo de violencia. Respecto al Perú, este estudio reveló que fue el tercer país en reportar una mayor prevalencia de violencia física o sexual a la mujer ( 15 a 49 años de edad) por parte de su pareja (casadas o en unión libre) alguna vez en los 12 meses precedentes.

A nivel nacional, el último estudio reportado por la Encuesta Demográfica y de Salud Familiar del Instituto Nacional de Estadística e Informática (INEI, 2012), indicó que el $66.3 \%$ de las mujeres alguna vez unidas (casadas, convivientes, divorciadas, separadas o viudas) manifestaron que el esposo o compañero ejerció alguna forma de control sobre ellas; el 21.7\% refirieron que habían experimentado situaciones de violencia verbal; el $19.9 \%$ declararon que habían sido amenazadas por su esposo o compañero con irse de la casa, quitarle a sus hijas(os) o retirarle la ayuda económica; mientras que el 37.2\% manifestaron que fueron víctimas de violencia física y sexual por parte de su esposo o compañero (como empujones, golpes, patadas, ataques o amenaza con cuchillo, pistola u otra arma, tener relaciones 
sexuales sin su consentimiento o realizar actos sexuales que la mujer no aprobaba).

Otras investigaciones refieren que las consecuencias de la violencia en la pareja recaen sobre las mujeres, generando ello daños significativos en su integridad. Así, en un estudio longitudinal con 375 mujeres, Watkins y colaboradores (2014) encontraron que tanto las agresiones físicas como psicológicas por parte de la pareja generan un impacto negativo en la salud física y mental de las mujeres, destacándose síntomas ansiosos, somáticos y depresivos.

García-Moreno y otros (2013) concluyeron que las mujeres que habían sido víctimas de abusos físicos o sexuales por parte de su pareja presentan mayor riesgo de padecer una serie de problemas de salud física como contraer sífilis, clamidia o gonorrea, así como mayor probabilidad de padecer disturbios mentales (como depresión) y son más propensas a tener problemas con el abuso del alcohol.

Por su parte, Bott y colaboradores (2014) reportaron que las mujeres que sufren violencia por parte de su esposo/compañero informaron haber resultado físicamente lesionadas, incluidas lesiones 'leves' como contusiones y dolores, así como lesiones más 'graves', como fracturas de huesos, quemaduras o heridas de cuchillo; extensas consecuencias emocionales y de salud mental, tales como temor, depresión, pensamientos suicidas, ansiedad o angustia suficientemente graves para no poder realizar su trabajo habitual.

En relación a la etiología, la tendencia actual señala la multicausalidad de la violencia de pareja, reconociendo la existencia de factores de riesgo que predisponen y mantienen el problema de la violencia a la mujer por la pareja. En este contexto, el modelo explicativo más utilizado por diferentes autores para la comprensión y atención de la violencia es el modelo ecológico planteado por Heise (1994), el cual señala que muchos factores de riesgo interactúan entre sí y facilitan la aparición de violencia de pareja. Estos factores se ubican en diferentes niveles de análisis y pueden ser individuales (las variables psicológicas personales están comprendidas en este nivel), relacionales, comunitarios y sociales/culturales (Butchart et al., 2011). 
Ahora bien, parece ser que en los últimos años, con la finalidad de reenfocar la atención en factores de tipo socioculturales como inequidad de género, sociedad patriarcal, actitudes machistas, consumo de alcohol por parte del agresor, etc., se ha relegado el revisar y profundizar algunos de los factores individuales, que corresponden a la dimensión psicológica de la mujer, variables que forman parte de su estructura y que probablemente anteceden a una situación de violencia.

En el contexto local, esta situación parece no ser ajena. Por ejemplo, según la recopilación que hizo Bardales (2012) de aquellos estudios registrados en el periodo 2006 al 2010 sobre violencia hacia la mujer en la relación de pareja (entre otros tipos de violencia familiar y sexual), se puede deducir que la mayoría de ellos se orientan a brindar información descriptiva o relacionada con variables situacionales, contextuales y socioculturales, principalmente. Tales son así variables estudiadas como edad de la mujer, nivel de instrucción, nivel socioeconómico, estado civil, consumo de alcohol de la pareja, roles de género, patriarcalismo, número de años de la relación, antecedentes de violencia en la familia de origen, entre otros.

No cabe duda que el contexto sociocultural influye en la aparición de esta problemática, pero la misma relevancia tiene el enfocar también aquellos factores individuales, aquellas características psicológicas que una mujer presenta al inicio de sus relaciones de pareja y que pueden configurar desde ya un factor de riesgo para que sea víctima de violencia de pareja.

De esta forma, algunos estudios han encontrado asociación entre el maltrato en las relaciones de pareja y las variables psicológicas (Miljánovich et al., 2013), y específicamente, algunas características de personalidad (Ballester \& Ventura, 2010; Dubugras \& Guevara, 2013; López, Moral de la Rubia, Díaz \& Cienfuegos, 2013; Preciado-Gavidia, Torres-Cendales \& Rey-Anacona, 2012; Picó-Alfonso, Echeburúa \& Martínez, 2008).

Congruente a lo expuesto, una de las variables psicológicas individuales que es importante estudiar en este contexto es la dependencia emocional a la pareja, la cual, para efectos del siguiente estudio, será 
entendida como un tipo de dependencia interpersonal o relacional genuina (Castelló, 2005; Moral \& Sirvent, 2008) y definida como la dimensión disfuncional de un rasgo de personalidad, que consiste en la necesidad extrema de orden afectiva que una persona siente hacia su pareja (Castello, 2005, 2012). En este sentido, y siguiendo a este autor, la persona afecta de dependencia emocional presenta un patrón de pensar, sentir y actuar más o menos estable en su constitución, denota comportamientos de sumisión y subordinación a la pareja, necesidad de acceso a la misma, miedo a que la relación se termine, priorización de la pareja, entre otras características disfuncionales.

En este contexto, Amor y Echeburúa (2010) identificaron la dependencia emocional hacia el agresor como una característica de tipo emocional en mujeres víctimas de violencia de pareja, la cual implicaría la vivencia de emociones contradictorias hacia esta (aproximación y rechazo), vínculo emocional basado entre la intermitencia entre el buen y el mal trato, enamoramiento intenso, sensación de no poder vivir sin él y miedo a ser abandonada.

Castelló (2005) refiere que la dependencia emocional explicaría la conducta de algunas mujeres víctimas de violencia de pareja cuando justifican las infidelidades y agresiones de su pareja, cancelan procesos legales (denuncias), incumplen las órdenes judiciales de alejamiento respecto a la pareja, regresan con el agresor creyendo que es posible que sus parejas tomen conciencia y cambien de actitud, y en otros casos, inician una nueva relación con características similares de malos tratos.

Hirigoyen (2006) afirma que la dependencia emocional es una consecuencia del dominio y la manipulación que se da en la pareja violenta, creándose una verdadera adicción al compańero que se explica por mecanismos neurobiológicos y psicológicos para evitar sufrir y obtener un cierto sosiego.

Deza (2012) refiere que uno de los factores asociados a la permanencia de la mujer en relaciones violentas es la percepción de amor romántico que tiene de las relaciones de pareja, lo cual implica depender del otro y adaptarse a él, postergando incluso lo propio; perdonar y justificar todo en nombre del amor; consagrarse al bienestar 
del otro; desesperar ante la sola idea de que la pareja se vaya, sintiendo que nada vale tanto como esa relación; pensar todo el tiempo en el otro, hasta el punto de no poder trabajar, estudiar, comer o dormir; idealizar a la otra persona, no aceptando la existencia de ningún defecto; sentir que cualquier sacrificio es poco si se hace por amor al otro, entre otras características. A todo ello se sumaría una baja autoestima o desvalorización.

Villegas y Sánchez (2013) realizaron un estudio para identificar las características de dependencia afectiva en un grupo de mujeres denunciantes víctimas de maltrato por su pareja. Encontraron que las características psicológicas más sobresalientes de dependencia afectiva en las participantes fueron expresión límite (percepción de la ruptura de pareja como algo catastrófico y expresiones impulsivas), miedo a la soledad (la soledad es vista como algo aterrador y se evita por todos los medios), ansiedad por separación (miedo ante la amenaza de ser abandonados y preocupación por la pérdida), así como modificación de planes (modifican su vida con tal de retener a su lado la pareja).

Es preciso señalar que si bien se observa en la comunidad científica un interés por estudiar la relación entre la dependencia emocional y la violencia de pareja, algunos estudios han empleado diversos términos y definiciones un tanto confusas, generales, distintas al constructo en cuestión para referirse a la variable dependencia emocional (adicción al amor, amor romántico, dependencia interpersonal, etc.).

En nuestro contexto, escasos y desactualizados estudios nacionales señalan esta relación, aunque erróneamente se ha utilizado el constructo dependencia psicológica (término genérico y exportado del ámbito de estudio de los trastornos por consumo de sustancias psicoactivas) para referirse a las características de la dependencia emocional, utilizando instrumentos no adecuados (no válidos, adaptadas de pruebas que miden dependencia psicológica a sustancias psicoactivas) para medir la variable de interés.

En congruencia con todo lo señalado anteriormente, el siguiente estudio tiene como objetivo identificar la relación entre dependencia 
emocional y violencia de pareja en una muestra de mujeres usuarias del servicio de psicología de un establecimiento de salud.

La importancia de realizar investigaciones como estas que vinculen la violencia a la mujer en su relación de pareja con una variable de la dimensión psicológica de la mujer, específicamente, la dependencia emocional, queda justificada por el aporte teórico sobre el conocimiento de estas dos variables, así como el potencial práctico de utilizar dichos resultados para emprender acciones de intervención viables y específicas, tanto en el tratamiento psicológico de la violencia de pareja, como en la prevención de este problema desde etapas tempranas de la relación, como el enamoramiento y/o noviazgo.

\section{Método}

La siguiente investigación utiliza un enfoque cuantitativo y emplea una estrategia asociativa comparativa (Ato, López \& Benavente, 2013).

\section{Participantes}

La muestra del estudio estuvo constituida por 51 mujeres usuarias del servicio de psicología del Hospital Ernesto Germán Guzmán Gonzáles, en el distrito y provincia de Oxapampa, departamento Pasco, con edades entre los 18 y 60 ańos, de diferente nivel socioeconómico, hablantes del castellano, con un nivel de comprensión lectora equivalente al sexto año de educación básica regular, que no hayan sufrido trastornos mentales o causados por enfermedad incapacitante y que tengan actualmente una relación de pareja heterosexual o, en su defecto, que la hayan tenido. Se dividió la muestra en dos grupos en función a la presencia o ausencia de violencia de pareja. Así, el primero estuvo conformado por 25 mujeres víctimas de violencia de pareja (psicológico, física y/o sexual), ya sean episodios actuales o episodios anteriores (hace no más de tres meses atrás según criterios de registro para casos de violencia familiar propuesto por el Ministerio de Salud del Perú Minsa). Este grupo recibió la etiqueta de muestra clínica con violencia 
de pareja. El segundo grupo estuvo constituido por 26 mujeres que no reportaron ni fueron identificadas como víctimas de violencia de pareja, recibiendo la etiqueta de muestra clínica sin violencia de pareja.

El tipo de muestreo empleado fue no probabilístico e incidental, puesto que se determinó con anterioridad las características de los sujetos de la muestra, siendo, en este caso, 51 mujeres usuarias del Servicio de Psicología del Hospital Ernesto Germán Guzmán Gonzáles en el distrito y provincia de Oxapampa, departamento de Pasco.

\section{Medidas e instrumentos de medición}

Las variables que conforman el estudio son la violencia a la mujer en la relación de pareja o violencia de pareja (VIP), la cual es una variable de tipo cualitativa, con una medición de escala nominal, con las siguientes categorías: 1) Con violencia de pareja (subcategorías o dimensiones: violencia psicológica, violencia física y violencia sexual) y 2) Sin violencia de pareja. Así también, la variable dependencia emocional es una variable de tipo cuantitativa continua, con una medición de escala de intervalo, pudiendo ser su puntuación directa transformada en variable categórica con cuatro valores: bajo, significativo, moderado y alto. Se utilizaron tanto las puntuaciones directas como las categorías de la variable. También se consideró algunas variables descriptivas tales como edad (cualitativa, nivel de medición de escala nominal, agrupados en cuatro rangos de años), grado de instrucción (cualitativa, escala ordinal, 7 categorías), ocupación (cualitativa, escala nominal, 4 categorías), tipo de relación de pareja (cualitativa, escala nominal, 3 categorías) y relación de pareja actual (cualitativa, escala nominal, con 2 categorías).

Para la detección de violencia por parte de la pareja, se utilizaron los cuestionarios "Ficha de Tamizaje de la Violencia Basada en Género" (Anexo A) y la "Ficha Multisectorial Violencia Familiar" (Anexo B), los cuales son instrumentos técnicos normativos indicados por el Ministerio de Salud del Gobierno del Perú para registrar casos de violencia familiar (la violencia de pareja es un tipo de ellos). El primero consiste en un cuestionario de tamizaje, mientras que el segundo instrumento 
se aplica en caso de que el tamizaje anterior haya sido positivo. Estos instrumentos proporcionan información sobre datos de la persona agredida (edad, sexo, estado civil, etc.), datos del/la agresor/a (edad, sexo, vínculo con la víctima, etc.), datos de la agresión (estado del agresor, tipo de violencia, medio utilizado, etc.), medidas tomadas por la víctima y seguimiento del caso. Se consideró solo algunos ítems en función a los objetivos del estudio, tales como la edad de la víctima, nivel de instrucción, ocupación, estado civil o estado de relación de pareja, y el tipo de violencia (física, psicológica y sexual).

Asimismo, se empleó el Inventario de Dependencia Emocional IDE (Aiquipa, 2012) (Anexo C). Esta prueba, creada y validada en el Perú, se basa en el modelo de los rasgos de personalidad y el modelo teórico de Castelló (2005). Está compuesta por 49 reactivos en escala tipo Likert de 5 alternativas, agrupados en siete factores: 1) miedo a la ruptura (MR), 2) miedo e intolerancia a la soledad (MIS), 3) prioridad de la pareja (PP), 4) necesidad de acceso a la pareja (NAP), 5) deseos de exclusividad (DEX), 6) subordinación y sumisión (SS), y 7) deseos de control y dominio (DCD). El instrumento obtuvo indicadores de confiabiliadad satisfactorios: $\alpha$ de Crombach igual a .96; coeficiente de correlación $r$ de Pearson, corregido mediante la fórmula Spearman-Brown igual a .91; así como evidencias de validez de constructo adecuados: índices de acuerdo con valores de .80 y 1.00 para más del 95\% de los reactivos (validez de contenido); validez factorial (reproducción de 7 factores que explicaron el 58.25\% de la varianza total, conteniendo ítems con saturaciones entre .40 y .70); y validez de criterio (diferencias de medias entre grupo clínico y no clínico significativa al 95\% de nivel de confianza). Además, cuenta con baremos apropiados al contexto.

Se realizó un estudio piloto con 130 sujetos (88 mujeres y 42 varones) para identificar la confiabilidad (mediante el método de consistencia interna) en una muestra de sujetos con caracterísitcas similares a la muestra de estudio. De esta forma, se obtuvo un coeficiente $\alpha=.97$. 


\section{Análisis de datos}

Se utilizó el programa estadístico SPSS en su versión 20.0 El análisis cuantitativo incluyó estadísticos descriptivos para la muestra (frecuencias y medidas de tendencia central); pruebas para verificar distribución de normalidad; prueba para comparar puntuaciones en muestras independientes ( $t$ de student para muestras independientes) y coeficiente de asociación no lineal eta. Para todos los casos de pruebas estadísticas se utilizó un nivel de significancia menor a .01.

\section{Procedimiento}

Entre los meses de enero y mayo de 2013 se recolectaron los datos de la muestra total que asistieron al servicio de psicología del establecimiento de salud. Luego, se identificaron los casos de aquellas mujeres que reportaron ser víctimas de violencia por parte de su pareja heterosexual a través de la entrevista y evaluación psicológica inicial. Se procedió a la aplicación de forma individual del Inventario de Dependencia Emocional - IDE, aun dentro del proceso de evaluación, pero en una fecha distinta. Paralelamente, se identificaron a las usuarias que asistieron a consulta por diversos motivos (no trastornos crónicos y en estado de crisis) y que fueron descartadas como víctimas de violencia por su pareja heterosexual. Se le aplicó también el IDE y se recolectó el resto datos demográficos. Luego, se procedió a la corrección y consolidación de la información de los datos, análisis y elaboración del reporte de investigación.

Los aspectos éticos que reune la presente investigación están en relación con los principios básicos contamplados por la American Psychological Association (2010) y el Código de Ética Profesional del Colegio de Psicólogos del Perú (s.f.).

De esta manera, los resultados obtenidos son producto de un proceso sistemático, crítico y empírico realizado por el autor. Por tanto, sus datos han sido obtenidos de una parte de la realidad, han sido derivados de la información de un grupo de personas a través de una técnica específica empleada y han sido analizados y discutidos meticulosamente. 
La protección del bienestar y dignidad de las participantes se garantizó con el consentimiento informado verbal y la confidencialidad. La primera medida implicó comunicar todas las características de la investigación, respetar la libertad del individuo para declinar su participación o para que se retire de la investigación una vez iniciada, así como explicar y/o aclarar dudas sobre otros aspectos del estudio. La confidencialidad de las participantes fue posible a través de la no publicación de sus datos personales en ninguna parte del escrito ni la utilización de los mismos en procesos ajenos al estudio. Ambas medidas de protección fueron referidas a las colaboradoras durante el proceso de evaluación en el establecimiento de salud. Todas ellas aceptaron las condiciones manifestadas y accedieron a que se utilice la información pertinente al estudio.

\section{Resultados}

La Tabla 1 reporta las características descriptivas de la muestra. Se observa que el $33.3 \%$ de las participantes tienen entre 30 a 39 ańos, lo que corresponde a la etapa de vida de adultez media. Asimismo, se observa que el $23.5 \%$ tienen un nivel de instrucción superior técnico con estudios culminados. Respecto a la ocupación, el 39.2\% de las participantes son empleadas públicas. En esta misma tabla también se observa que el $45 \%$ de las participantes tienen una relación de convivencia con sus parejas y el $64.7 \%$ de la muestra tiene actualmente una relación de pareja heterosexual.

En la Tabla 2 se aprecia la frecuencia de mujeres víctimas de violencia de pareja, representando el $49 \%$ del total. Entre las que sufren de violencia de pareja, ninguna reportó violencia de tipo sexual, siendo más frecuente el tipo de violencia física (la cual implica algún tipo de violencia psicológica). Tomando como referencia esta distribución se conformaron los dos tipos de submuestras: con violencia de pareja (muestra clínica de casos) y sin violencia de pareja (muestra de comparación o de control). 


\section{Tabla 1}

Caracteristicas demográficas de la muestra total

\begin{tabular}{llcr}
\hline Variables & Categorías & frec & \multicolumn{1}{c}{$\%$} \\
\hline Edad $(M=32.2 ; D E=8.8)$ & 18 a 24 años & 11 & $21.6 \%$ \\
& 25 a 29 años & 11 & $21.6 \%$ \\
& 30 a 39 años & 17 & $33.3 \%$ \\
& 40 a 59 años & 12 & $23.5 \%$ \\
\hline Grado de Instrucción & Prim. Completa & 1 & $2.0 \%$ \\
& Sec. Completa & 10 & $19.6 \%$ \\
& Sec. Incompleta & 2 & $3.9 \%$ \\
& Sup. Téc. Completo & 13 & $25.5 \%$ \\
& Sup. Téc. Incompleto & 6 & $11.8 \%$ \\
& Sup. Univ. Completo & 11 & $21.6 \%$ \\
& Sup. Univ. Incompleto & 8 & $15.7 \%$ \\
\hline Ocupación & Estudiante & 5 & $9.8 \%$ \\
& Labores domésticas & 17 & $33.3 \%$ \\
& Empleada pública & 20 & $39.2 \%$ \\
& Independiente & 9 & $17.6 \%$ \\
\hline Tipo de relación de pareja & No conviviente & 14 & $27.5 \%$ \\
& Convivente & 23 & $45.0 \%$ \\
& Exconvivente & 14 & $27.5 \%$ \\
\hline & Sí Actual & 33 & $64.7 \%$ \\
& No & 18 & $35.3 \%$ \\
\hline
\end{tabular}


Dependencia emocional en mujeres víctimas de violencia de pareja / Aiquipa Tello

\section{Tabla 2}

Distribución de la muestra según la variable violencia de pareja (VIP)

\begin{tabular}{llrr}
\hline Categorías & Subcategorías & frec & $\%$ \\
\hline Sin violencia de pareja & & 26 & 51.0 \\
\hline Con violencia de pareja & Violencia Psicológica & 11 & 21.5 \\
& Violencia Física y Psicológica & 14 & 27.5 \\
& Violencia Sexual & 0 & .0 \\
& Total & 51 & 100.0 \\
\hline
\end{tabular}

En la Tabla 3 se observa las categorías de dependencia emocional según el tipo de muestra (con violencia de pareja y sin violencia de pareja). Así, el 96\% de la muestra clínica de casos, obtuvo la categoría de alto, mientras que en la muestra de comparación, solo el 19\% obtuvo esa categoría, en tanto que el $42 \%$ obtuvo la categoría bajo o normal.

\section{Tabla 3}

Categorias de Dependencia Emocional (Escala total) según el tipo de muestra

\begin{tabular}{lcccccc}
\hline & \multicolumn{4}{c}{ Tipo de Muestra } & \multirow{2}{*}{ Total } \\
\cline { 2 - 5 } Categorías & $\begin{array}{c}\text { Con violencia de } \\
\text { pareja }\end{array}$ & $\begin{array}{c}\text { Sin violencia de } \\
\text { pareja }\end{array}$ & & \\
\cline { 2 - 6 } & frec & $\%$ (muestra) & frec & $\%$ (muestra) & frec & $\%$ \\
\hline Alto & 24 & $96.0 \%$ & 5 & $19.3 \%$ & 29 & $56.9 \%$ \\
Moderado & 0 & $.0 \%$ & 3 & $11.5 \%$ & 3 & $5.9 \%$ \\
Significativo & 0 & $.0 \%$ & 7 & $26.9 \%$ & 7 & $13.7 \%$ \\
Bajo o Normal & 1 & $4.0 \%$ & 11 & $42.3 \%$ & 12 & $23.5 \%$ \\
\hline Total & 25 & $49 \%$ & 26 & $51 \%$ & 51 & $100.0 \%$ \\
\hline
\end{tabular}


La Tabla 4 muestra la bondad de ajuste a una distribución normal de la variable dependencia emocional. Se denotan las puntuaciones medias (escala total y dimensiones) de dependencia emocional y el nivel de significancia del valor de la Prueba Kolmogorov-Smirnov, siendo esta mayor a .01 para todos los casos.

\section{Tabla 4}

Prueba de ajuste de Normalidad para la variable dependencia emocional (escala total y dimensiones)

\begin{tabular}{lccccc}
\hline Dependencia Emocional & $\mathrm{N}$ & $M$ & $D E$ & $\mathrm{~K}-\mathrm{S}$ & $p$ \\
\hline Escala total & 51 & 139.5 & 56.3 & .94 & .35 \\
Miedo a la ruptura (MR) & 51 & 23.6 & 11.7 & 1.05 & .22 \\
Miedo e intolerancia a la soledad (MIS) & 51 & 31.6 & 13.7 & 1.24 & .09 \\
Prioridad de la pareja (PP) & 51 & 21.3 & 10.6 & 1.03 & .24 \\
Necesidad de acceso a la pareja (NAP) & 51 & 18.2 & 6.8 & .87 & .43 \\
Deseos de exclusividad (DEX) & 51 & 13.8 & 6.5 & 1.11 & .17 \\
Subordinación y sumisión (SS) & 51 & 15.7 & 6.1 & .69 & .71 \\
Deseos de control y dominio (DCD) & 51 & 15.3 & 5.9 & .68 & .72 \\
\hline
\end{tabular}

${ }^{*}$ Nota: Las distribuciones de la escala total y factores son normales; $p<.01$

La Tabla 5 presenta las diferencias de medias en los puntajes de dependencia emocional (escala total y dimensiones), así como los valores de la prueba $t$ de student para muestras independientes, siendo sus niveles de significancia menores a .01 .

Por último, la Tabla 6 ofrece el coeficiente de asociación eta para las variables de estudio, el cual asume valores elevados cuando la variable violencia de pareja asume el rol de variable dependiente. También se presenta el coeficiente eta al cuadrado. 
Dependencia emocional en mujeres víctimas de violencia de pareja / Aiquipa Tello

\section{Tabla 5}

Diferencia entre muestra clínica de casos y muestra de comparación

\begin{tabular}{lcccccccc}
\hline \multirow{2}{*}{$\begin{array}{l}\text { Dependencia } \\
\text { Emocional }\end{array}$ (Escala Total/ } & \multicolumn{9}{c}{ Tipo de Muestra } & \multicolumn{2}{c}{$\begin{array}{l}\text { Con violencia de } \\
\text { pareja }(\mathrm{n}=25)\end{array}$} & \multicolumn{2}{c}{$\begin{array}{l}\text { Sin violencia de } \\
\text { pareja }(\mathrm{n}=26)\end{array}$} & & & & \\
\cline { 2 - 6 } Factores) & $M$ & $D E$ & $M$ & $D E$ & & & & \\
viol & $-M_{\text {sinviol }}$ & $t$ & $p$ \\
\hline Escala Total & 183.6 & 39.5 & 97 & 32 & 86.6 & 8.6 & .000 \\
MR & 33.6 & 7.5 & 14 & 5 & 19.6 & 11 & .000 \\
MIS & 41.4 & 11.1 & 22 & 7.9 & 19.4 & 7.2 & .000 \\
PP & 29.2 & 7.8 & 13.7 & 6.7 & 15.5 & 7.7 & .000 \\
NAP & 21.8 & 6.4 & 14.8 & 5.3 & 7 & 4.3 & .000 \\
DEX & 17.9 & 5.8 & 9.8 & 4.5 & 8.1 & 5.6 & .000 \\
SS & 20.3 & 4 & 11.4 & 4.1 & 8.9 & 7.8 & .000 \\
DCD & 19.4 & 4.5 & 11.4 & 4.1 & 8 & 6.7 & .000 \\
\hline
\end{tabular}

${ }^{*}$ Nota: $p<.01$

\section{Tabla 6}

Medida de asociación entre variables de estudio

\begin{tabular}{|c|c|c|c|}
\hline \multirow[b]{2}{*}{ Escala Total/Factores } & \multicolumn{2}{|c|}{ Coeficiente de asociación Eta } & \multirow[b]{2}{*}{$\begin{array}{c}\text { Eta } \\
\text { cuadrado }\end{array}$} \\
\hline & $\begin{array}{c}\text { Escala total/ } \\
\text { Factores dependiente }\end{array}$ & $\begin{array}{l}\text { Tipo de Muestra } \\
\text { dependiente }\end{array}$ & \\
\hline $\mathrm{DE}^{*}$ Tipo de Muestra & .78 & .94 & .60 \\
\hline MR ${ }^{*}$ Tipo de Muestra & .84 & .93 & .71 \\
\hline MIS * Tipo de Muestra & .72 & .86 & .51 \\
\hline $\mathrm{PP}^{*}$ Tipo de Muestra & .74 & .91 & .55 \\
\hline $\mathrm{NAP}^{*}$ Tipo de Muestra & .52 & .76 & .27 \\
\hline DEX $*$ Tipo de Muestra & .63 & .82 & .39 \\
\hline SS * Tipo de Muestra & .75 & .89 & .56 \\
\hline DCD * Tipo de Muestra & .69 & .88 & .48 \\
\hline
\end{tabular}




\section{Discusión}

Los resultados sugieren que existe relación estadísticamente significativa entre la variable dependencia emocional (variable que representa un factor de tipo individual y psicológico) y la variable violencia de pareja, puesto que se encontraron diferencias significativas entre la muestra clínica con violencia de pareja (grupo de casos) y la muestra clínica sin violencia de pareja (grupo de comparación), siendo las participantes que viven o han vivido violencia de pareja las que obtuvieron puntuaciones mucho más elevadas de dependencia emocional respecto a aquellas mujeres que no han vivido tal experiencia. Los valores que asumieron el coeficiente de relación eta en la puntuación general de dependencia emocional y en sus dimensiones así lo confirman. Algunos estudios como los de Amor y Echeburúa (2010); Castelló (2005) y Villegas y Sánchez (2013) encontraron resultados similares.

Se observa también, de forma general, que el $60 \%$ de la varianza de dependencia emocional es explicada por la variable violencia de pareja (los valores del coeficiente de relación eta cuadrado son elevados para la escala total y para cada uno de los factores).

Asimismo, se deduce empíricamente que tres de las dimensiones o factores de la dependencia emocional se encuentran más relacionados con la violencia de pareja, puesto que sus coeficientes de asociación tuvieron valores más elevados (coeficiente eta con valores entre .89 a .92). Estas son: miedo a la ruptura, prioridad de pareja y subordinación y sumisión.

De esta forma, una mujer con dependencia emocional experimentará elevados niveles de miedo al pensar que su relación de pareja pueda terminar. Por ello es que evitará por todos los medios que eso se cumpla y es posible que llegue a tolerar conductas que van de simples y esporádicas desatenciones por parte de su pareja hasta insultos y ofensas reiterativas, incluso agresiones físicas. Si en algún momento su pareja decide terminar la relación, aquella rogará para que el otro recapacite su decisión, suplicará que le dé una nueva oportunidad, pedirá perdón por faltas que no cometió y se atribuirá toda la responsabilidad para 
que la relación de pareja no acabe. Mostrará resistencia a terminar esa relación, y si aun así se da la ruptura, mantendrá comunicación directa o indirecta con la pareja (revisará las redes sociales, preguntará a las amistades de la pareja sobre su situación amorosa, hablará con los familiares de aquel para que aboguen por ella, etc.).

Por otra parte, el hecho de que una mujer priorice a su pareja sobre cualquier otra actividad, interés o persona (familiares, amigos, ella incluso), garantiza una relación desequilibrada. Cuando la mujer considera que su pareja es más importante que cualquier persona o cosa, empodera al otro y le confiere permisos que pueden perjudicar la relación y su propio bienestar. Así, se va construyendo un sistema de creencias en las que se concibe a la pareja como omnipotente y ella se etiqueta como la incompetente e indigna del otro.

Estas condiciones, en relación también a las otras características de la dependencia emocional, facilitan que la mujer asuma un rol sumiso y se subordine a su pareja. En este contexto, la mujer acatará cuanto la pareja le diga, sin evaluar cognitivamente dichas peticiones (u órdenes). Sus intereses estarán en segundo lugar y es probable que ni se tomen en cuenta. No tendrá derecho a opinar sobre algún aspecto de la relación, ni tendrá la libertad de expresar lo que le incomode del otro. Paulatinamente se va instalando en la relación prohibiciones, vejaciones, humillaciones y aceptación incondicional, lo que ya constituye maltrato psicológico. A partir de lo mencionado, se abre paso a la violencia física, se consolida la subordinación y sumisión, la dependencia emocional se cronifica y se afianza la violencia de pareja. Por supuesto, este actuar por parte de la mujer que vive una relación así, incluso ya con el maltrato, no es una expresión de rasgos masoquistas, como se atribuía en algunas disertaciones, sino más bien, como dice Castelló (2005), la sumisión representa para la persona dependiente un medio para que la relación no termine, puesto que hay miedo a la ruptura, que en última instancia revela esa fuerte necesidad de vinculación afectiva a la pareja que es el sustento de la dependencia emocional.

Si bien se encontró que existe una fuerte relación entre las variables de estudio, y aunque no se afirma categóricamente que la dependencia 
emocional se comporte como un factor de riesgo para que la mujer tolere maltrato por parte de la pareja, los resultados sugieren esa dirección, puesto que el coeficiente de asociación eta asume un valor alto cuando la violencia de pareja se comporta como variable dependiente. Sin duda, esto es congruente con algunos planteamientos y estudios que se han aproximado al papel que tiene la dependencia emocional en el inicio y mantenimiento de violencia de pareja (Amor \& Echeburúa, 2010; Castelló, 2005). Además, si se tiene en cuenta que el modelo de dependencia emocional del presente estudio está basado en la teoría de los rasgos y en el modelo de Castelló (2005), congruente al primero, la dependencia emocional configuraría un conjunto de emociones, pensamientos y conductas más o menos estables en la persona que la padece, y por lo tanto, la mujer que sufre violencia de pareja tiene como parte de su estructura de personalidad ese rasgo, el mismo que ha antecedido a su relación de pareja y por ende a las experiencias de violencia en ese contexto.

Aunque el siguiente estudio ha utilizado una muestra pequeña en función a la disposición de participantes, y como tal puede argumentarse que no es representativa y por tanto no generalizable, se cree que los resultados que se han obtenido contribuyen en alguna medida a la comprensión de la mujer violentada por su pareja. Desde este punto de vista, la relación hallada entre la dependencia emocional y la violencia de pareja en la mujer constituye datos empíricos - y no simples conjeturas de sentido común como es frecuente en nuestro medio- que pueden aportar alternativas de intervención psicológicas en la prevención y tratamiento de mujeres víctimas de violencia de pareja.

Así, las actividades de prevención y promoción, principalmente en la población adolescente y joven, han de incidir en las consecuencias de depender emocionalmente de la pareja y cómo esta característica permite tolerar actitudes y conductas de violencia, justificándose estos comportamientos por el supuesto "amor incondicional que se ha de tener a la pareja” (entre otras creencias individuales y sociales), lo cual precipita nuevamente ciclos de violencia cada vez más severos que probablemente, si no se hace algo al respecto, conlleve a otro caso más de 
feminicidio. En este sentido, es conveniente que se trabaje con grupos de mujeres con dependencia emocional.

Respecto al tratamiento, las intervenciones psicológicas con las mujeres afectas de violencia de pareja que presentan dependencia emocional, han de seguir programas de tratamiento específicos que contemplen las dimensiones de la dependencia emocional, puesto que si no se trabaja esos aspectos, es probable que retomen la relación con el agresor o inicien nuevas relaciones de pareja con características similares de maltrato. En este contexto, el Ministerio de Salud del Gobierno Peruano tiene la responsabilidad de contemplar dentro de sus programas de atención a mujeres víctimas de violencia de pareja estos aspectos con la finalidad de recuperar la salud mental de la mujer.

Queda pendiente seguir investigando más sobre la relación entre la dependencia emocional y la violencia de pareja en la mujer en nuestro contexto en muestras más grandes y de diferentes procedencias, desde otro enfoque de investigación (cualitativo o mixto), y por supuesto, sobre la base de esos descubrimientos y no por una simple exportación de prácticas extranjeras descontextualizadas, proponer alternativas eficaces de intervención para este problema.

\section{Referencias}

Aiquipa, J. (2012). Diseño y Validación del Inventario de Dependencia Emocional - IDE. Revista de Investigación en Psicología, Facultad de Psicología, UNMSM, 15(1), 133-145.

American Psychological Association. (2010). American Psychological Association ethical principles of psychologists and code of conduct. Recuperado de http://www.apa.org/ethics/code/index.aspx Amor, P. \& Echeburúa, E. (2010). Claves Psicosociales para la permanencia de la víctima en una relación de maltrato. Clínica Contemporánea, 1(2), 97-104.

Ato, M., López, J. \& Benavente, A. (2013). Un sistema de clasificación de los diseños de investigación en psicología. Anales de Psicología, 29(3), 1038-105. 
Ballester, A. \& Villanueva, L. (2014). Valoración psicológica en delitos de violencia de género mediante el Inventario Clínico y Multiaxial de Millon III (MCMI-III). Anuario de Psicología Jurídica, 24, 9-18.

Bardales, O. (2012). Estado de las investigaciones en violencia familiar y sexual 2006-2010. Lima: Ministerio de la Mujer y Población Vulnerable (MIMP).

Bott, B., Guedes, A., Goodwin, M. \& Adams, J. (2014). Violencia contra las mujeres en América Latina y el Caribe: análisis comparativo de datos poblacionales de 12 paises. Washington, DC: Organización Panamericana de la Salud.

Butchart, A., García-Moreno, C. \& Mikton, C. (2011). Prevención de la violencia sexual y violencia infligida por la pareja contra las mujeres: qué hacer y cómo obtener evidencias. Washington, DC: Organización Panamericana de la Salud.

Castelló, J. (2005). Dependencia Emocional: Caracteristicas y Tratamiento. España: Alianza Editorial.

Castelló, J. (2012). La Superación de la Dependencia Emocional. Málaga: Ediciones Corona Borealis.

Colegio de Psicólogos del Perú (s.f.). Código de Ética Profesional. Recuperado de http://www.colegiodepsicologosperu.org/w/ imagenes/enlaces/archivos/CodigoEticaPeru.pdf.

Deza, S. (2012). ¿Por qué las mujeres permanecen en relaciones de violencia? Avances en Psicología, 20(1), 45-55.

Dubugras, S. \& Guevara, B. (2013). Personalidade de mulheres vítimas de violencia doméstica: uma revisão sistemática da literatura. Contextos Clínicos, 6(2), 106-116. doi:10.4013/ctc.2013.62.04.

García-Moreno, C., Henrica A., Watts, Ch., Ellsberg, M. \& Heise, L. (2005). Estudio multipais de la OMS sobre salud de la mujer y violencia doméstica contra la mujer. Ginebra: Organización Mundial de la Salud.

García-Moreno, C., Pallitto, C., Devries, K., Stockl, H., Watts, Ch. \& Abrahams, N. (2013). Estimaciones mundiales y regionales de la violencia contra la mujer: prevalencia y efectos de la violencia con- 
yugal y de la violencia sexual no conyugal en la salud. Resumen de orientación. Ginebra: Organización Mundial de la Salud.

Heise, L. (1994). Violencia contra la mujer. La cara oculta de la salud.

Washington: Programa Mujer, Salud y Desarrollo, Organización Panamericana de la Salud (OPM).

Heise, L. \& García-Moreno, C. (2003). La violencia en la pareja. En

E. Krug, L. Dahlberg, J. Mercy, A., Zwi \& R. Lozano (Eds.), Informe mundial sobre la violencia y la salud (pp. 97-131).

Washington, D.C.: Organización Panamericana de la Salud.

Hirigoyen, M. (2006). Mujeres maltratadas. Los mecanismos de la violencia en la pareja. Barcelona: Paidós.

Instituto Nacional de Estadística e Informática (2012). Encuesta Demográfica y de Salud Familiar 2012. Lima.

López, F., Moral de la Rubia, J., Díaz, R. \& Cienfuegos, Y. (2013).

Violencia en la pareja. Un análisis desde una perspectiva ecológica. Ciencia Ergo Sum, 20(1), 6-16.

Miljánovich, M., Huerta R., Campos, E., Torres, S., Vásquez, V., Vera, K. \& Díaz, G. (2013). Violencia familiar: modelos explicativos del proceso a través del estudio de casos. Revista de Investigación en Psicología, 16(1), 29-44.

Ministerio de la Mujer y Población Vulnerable (09 de octubre de 2013). Municipios deben implementar casas refugio para víctimas de violencia contra la mujer [Nota de prensa]. Recuperado de http://www. mimp.gob.pe/index.php?option=com_content\&view=article \&id=4133:municipios-deben-implementar-casas-refugio-paravictimas-de-violencia-contra-la-mujer\&catid $=54 \&$ Itemid $=445$

Moral, M. \& Sirvent, C. (2008). Dependencias sentimentales o afectivas: etiología, clasificación y evaluación. Revista Española de Drogodependencias, 33(2), 150-167.

Picó-Alfonso, M., Echeburúa, E. \& Martínez, M. (2008). Personality disorder symptoms in women as a result of chronic intimate male partner violence. Journal of Family Violence, 23, 577-588.

Preciado-Gavidia, P., Torres-Cendales, N. \& Rey-Anacona, A. (2012). Mujeres que finalizaron una relación maltratante: características 
de personalidad, psicopatológicas y sociodemográficas. Universitas Psychológica, 11(1), 43-54.

Villegas, M. \& Sánchez, N. (2013). Dependencia emocional en un grupo de mujeres denunciantes de maltrato de pareja. Revista Textos-\&-Sentidos, 7, 10-29.

Watkins, L., Jaffe, A., Hoffman, L., Gratz, K., Messman-Moore, T. \& DiLillo, D. (2014). The longitudinal impact of intimate partner aggression and relationship status on women's physical health and depression symptoms. Journal of Family Psychology, 28(5), 655-665.

Recibido: 06 de febrero, 2015

Aceptado: 20 de marzo, 2015 
Dependencia emocional en mujeres víctimas de violencia de pareja / Aiquipa Tello

\section{Anexo A}

Ficha de Tamizaje de la Violencia Basada en Género

GUIA TÉCNICA PARA LA ATENCIÓN INTEGRAL DE LAS PERSONAS AFECTADAS POR LA VIOLENCIA BASADA EN GENERO

\section{ANEXO II}

\section{FICHA DE TAMIZAJE}

DIRECCION DE SALUD:

ESTABLECIMIENTO.

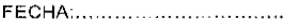

SERVICIO: Emergencia $\square$ Pediatria $\square$ Ginecologia $\square$ Obstetricia $\square$ CRED. $\square$ Medicina $\square$ P.F. $\square$ Otros

NOMBRE Y APELLIDO DEL USUARIO

EDAD:

SEXO: Masculino

Femenino

DIRECCION

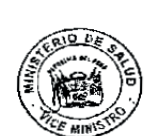

Debido a que la Violencia familiar es dañina para la salud de las personas, en todos los programas de salud estamos

preguntando a los pacientes si actualmente están en ésta situación para participar con ellas en la solución de su problema, por favor, conteste a estas preguntas:

\section{Fernàndez E.}

-

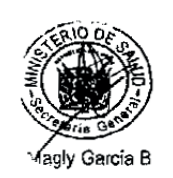

Sí es adulto (a):

Lalguna vez ha sido maltratada psicológicamente? ¿aiguna vez ha sido maltratada físicamente? ¿ha sido forzada a tener relaciones sexuales?

\section{Pregunte:}

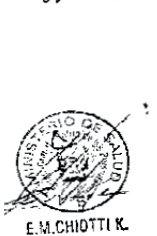

Si es niño, niña - adolescente:

¿Te castigan tu papá o tu mamá? ¿Como?

¿Alguna persona extraña o de tu propia familia te ha tocado de manera desagradable?

Marque con aspa $(\bar{x})$ todos los Indicadores de maltrato, que observe

En todos los casos: niña (o), adolescente, adulto(s), adulta mayor( 0 )

\begin{tabular}{|c|c|c|}
\hline FISICOS & Retraimiento & Intento de suicidio \\
\hline $\begin{array}{l}\text { Hematomas y contusiones } \\
\text { inexplicables }\end{array}$ & Lianto frecuente & Uso de alcshol, drogas \\
\hline Cicatrices o quemaduras & $\begin{array}{l}\text { Fxagerada necesidad de ganar, } \\
\text { sobresalir. }\end{array}$ & Tranquilizantes o analgésicos. \\
\hline Fracturas inexplicables & Demandas excesivas de atención. & SEXUALES \\
\hline Marca de mordeduras. & $\begin{array}{l}\text { Mucha agresividad o pasividad frental } \\
\text { a otros niños. }\end{array}$ & $\begin{array}{l}\text { Conocimiento y conducta sexua } \\
\text { inapropiadas (niños) }\end{array}$ \\
\hline $\begin{array}{l}\text { Lesiones de vulva. perineo y } \\
\text { recto, etc. }\end{array}$ & Tartamudeo & $\begin{array}{l}\text { Irfitación, dolor, lesión y } \\
\text { hemorragia en zona genital. }\end{array}$ \\
\hline
\end{tabular}

C. Mantilia P.

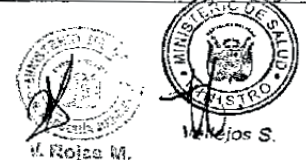




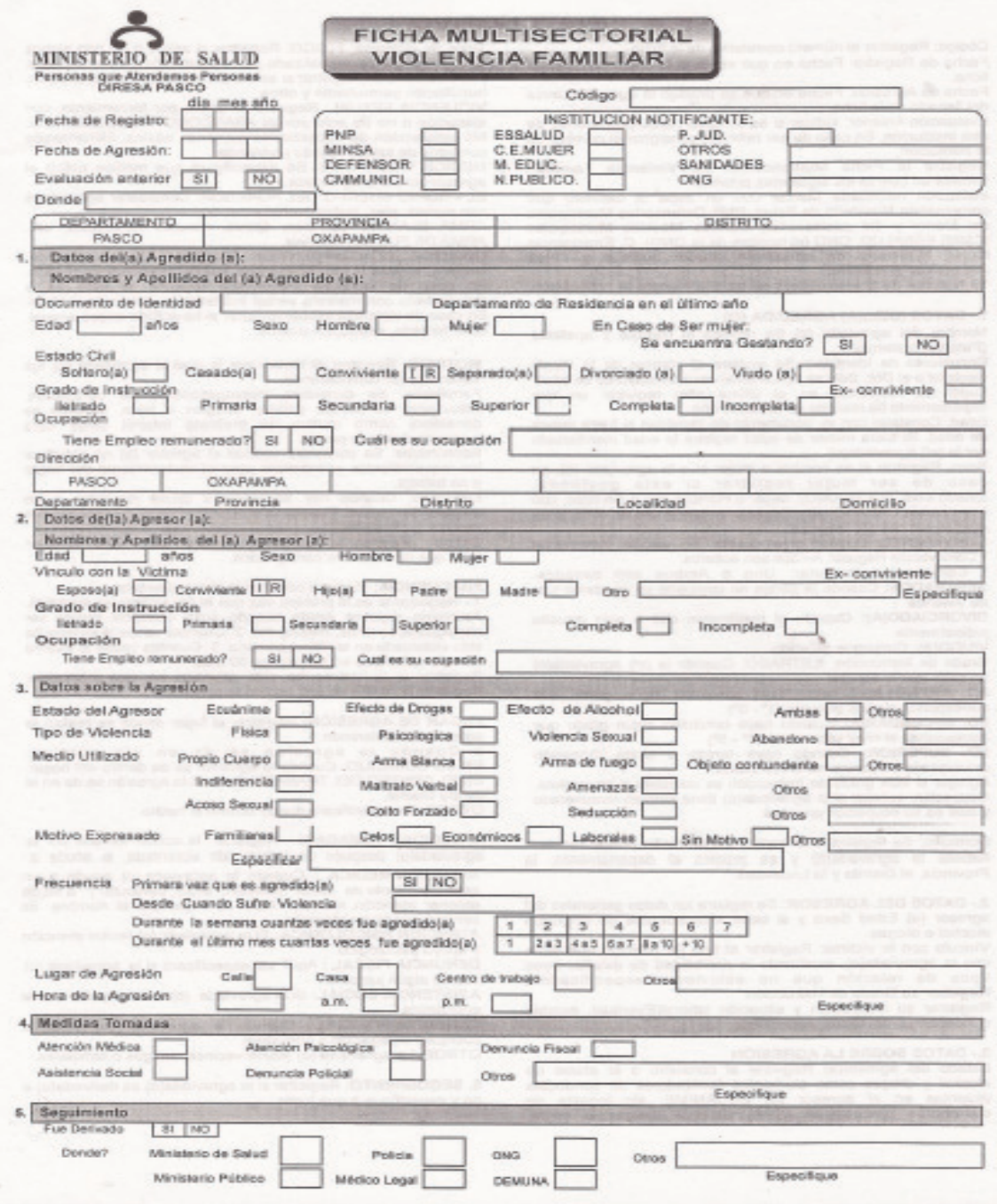


Ficha Multisectorial Violencia Familiar

Ficha Técnica del Inventario de Dependencia Emocional - IDE

Nombre:

Autor:

Procedencia:

Inventario de Dependencia Emocional - IDE. Jesús Joel Aiquipa Tello.

Facultad de Psicología - Universidad Nacional Mayor de San Marcos, Lima-Perú.

Fecha de Construcción: Marzo 2010.

Ámbito de Aplicación: Adultos, varones y mujeres de 18 años a más. Administración: Individual y Colectiva.

Duración: Entre 20 y 25 minutos.

Finalidad: El Inventario de Dependencia Emocional IDE es un instrumento objetivo de dominio específico que mide la dependencia emocional. Posee 7 factores: 1) Miedo a la ruptura, 2) Miedo e Intolerancia a la soledad, 3) Prioridad de la pareja, 4) Necesidad de acceso a la pareja, 5) Deseos de exclusividad, 6) Subordinación y sumisión, y 7) Deseos de control y dominio.

Interpretaciones: $\quad$ Se utilizan baremos percentilares, tanto para la puntuación total como para cada factor. Asimismo, presenta cuatro categorías diagnósticas: Bajo o Normal, Significativo, Moderado y Alto. 